\title{
Estudio De La Brecha Empresario-Investigador. La Percepción Del Investigador
}

\author{
Carlos Ángel Méndez-Peón, M.C.
}

Universidad Popular Autónoma del Estado de Puebla, Universidad de Sonora Estudiante de Doctorado en Planeación Estratégica y Dirección de

Tecnologías.

Margarita Herrera-Avilés, PhD

Instituto Tecnológico y de Estudios Superiores de Monterrey, campus Puebla.

\section{Alfredo Toriz-Palacios, PhD \\ Manuel González-Pérez, PhD, SNI I}

Universidad Popular Autónoma del Estado de Puebla

doi: 10.19044/esj.2016.v12n28p109 URL:http://dx.doi.org/10.19044/esj.2016.v12n28p109

\begin{abstract}
Technological innovation drives competitiveness and productivity of nations. The university-enterprise relationship (RUE) is determinant for the generation of an efficient innovation. The entrepreneur-researcher binomial (E-R) is the core process research and development (R \& D) and technology transfer and knowledge (TTK). Despite efforts to improve RUE, little has been done to strengthen the relationship between E-R. This study confirms the existence of a gap between entrepreneur and researcher (ERG), it allows us to understand the importance of empathy in collaboration and identify factors affecting the relationship. The data were obtained by the exploratory and transversal approach, through interviews and questionnaires Likert. The sample consisted of expert researchers who have participated in joint innovation projects with entrepreneurs. The results indicate that $75 \%$ of researchers surveyed perceive the existence of an ERG under collaborative $\mathrm{R}$ \& D and TTK. 78\% of scientists see empathy as a significant variable for joint projects. The study revealed that only $16 \%$ of the factors affecting RUE influence the relationship of the binomial E-I significantly.
\end{abstract}

Keywords: Entrepreneur-Researcher Gap (ERG), Technological Innovation, University-Enterprise Relationship (RUE) Competitiveness, Strategy, R \& D, TTK 


\section{Resumen}

La innovación tecnológica impulsa la competitividad y la productividad de las naciones. La relación universidad-empresa (RUE) es determinante para la generación de un sistema de innovación eficiente. El binomio empresario-investigador (E-I) es el núcleo del proceso de investigación y desarrollo ( + D) y de transferencia de tecnología y conocimiento (TTC). A pesar de los esfuerzos para mejorar la RUE, poco se ha hecho para fortalecer la relación entre E-I. Este estudio confirma la existencia de una brecha entre empresario e investigador (BEI), permite entender la importancia de la empatía en la colaboración entre E-I e identificar los factores que afectan a la relación entre ambos. Los datos fueron obtenidos por el enfoque exploratorio y transversal, a través de entrevistas y cuestionarios tipo Likert. La muestra consistió en investigadores expertos que han participado en proyectos conjuntos de innovación con empresarios. Los resultados indican que el 75\% de los investigadores encuestados perciben la existencia de una BEI en el marco de colaboración para la I + D y TTC. El 78\% de los investigadores ven la empatía como una variable significativa para los proyectos conjuntos. El estudio reveló que sólo el 16\% de los factores que afectan las RUE influyen en la relación del binomio E-I significativamente.

Palabras clave: Brecha Empresario Investigador (BEI), Innovación Tecnológica, Relaciones Universidad Empresa (RUE), Competitividad, Estrategia, I+D, TTC

\section{Introducción}

La competitividad es la condición determinante de la productividad de un país. A su vez, la productividad establece el nivel de prosperidad, crecimiento y calidad de vida de cualquier nación, pero adquiere mayor relevancia para los países emergentes y en vías de desarrollo en el nuevo mundo globalizado.

La innovación tecnológica se considera el pilar de mayor valor estratégico para fortalecer y apuntalar la productividad y la competitividad de una nación, debido a que es el único que puede asegurar un aumento sostenido en el nivel de prosperidad de vida en el largo plazo, puesto que su rendimiento no decrece con el tiempo (World Economic Forum, 2011). La relación o colaboración entre las universidades y centros de investigación con las empresas o industria (RUE) es una variable clave para robustecer al pilar y el sistema de innovación.

Aun cuando la innovación tecnológica se ha vuelto un proceso colectivo (Radu, 2015), una amplia y estrecha colaboración entre universidades e industria es altamente significativa (World Economic Forum, 
2011). Entre ambas organizaciones pueden producir nuevos bienes, procesos y conocimientos, estos productos impulsan mejor la investigación y desarrollo (I+D) y genera progreso tecnológico (Chen \& Dahlman, 2005). Esta colaboración, a su vez, aumenta el patentamiento y resuelve problemas de base tecnología para la sociedad. Sin embargo, la RUE ha tenido una trayectoria azarosa y mantiene todavía muchas barreras y pocas explicaciones (Bruneel, D'esteb \& Salter, 2010).

Los actores del sistema de innovación han tenido un interés creciente por mejorar la interacción entre la universidad y la industria en las ultimas decadas (Azagra-Caro, 2007). El gran valor visto para la economía y la insuficiente literatura actual, son motivadores para las investigaciones en diversas líneas y en varios países (Bruneel, et al., 2010). Se han abordado hasta nuestros días aspectos tan diversos, como los mostrados en la Tabla 1.

Tabla 1. Aspectos diversos de las RUE abordados y sus autores

Aspectos de las RUE

La participación del gobierno en las RUE.

La colaboración entre universidad y las PYMES. Los canales de colaboración.

El comportamiento innovador de las empresas. Las oficinas de transferencia de tecnología (OTT) y su importancia para las RUE.

La situación geográfica de las empresas y la

frecuencia, rendimiento y movilidad de los investigadores.

Las prácticas empresariales para las RUE. Los beneficios de hacer I + D con las universidades.

El impacto y los factores en la vinculación universidad-industria.

Las RUE y la innovación en industrias maduras y emergentes de países en desarrollo y países avanzados.

La estructuración de la teoría de colaboración universidad-industria.

La calidad de las relaciones universidad empresa. La distancia cognitiva. El capital social.

La naturaleza de los obstáculos.

Las diferencias culturales.

Los lazos entre académicos e industriales.
AUTOR(ES)

(Etzkowitz \& Leydesdorff, 2000), (Inzelt, 2004), (Sapién-Aguilar, Piñón-Howlet, \& Gutiérrez-Diez, 2015).

(Motohashi, 2005), (Bjerregaard, 2010) (Bekkers \& Bodas Freitas, 2008), (Fernández, et al., 2010), (Dutrenit \& Arza, 2010), (De

Fuentes y Dutrenit, 2012)

(Shichijo, Baba \& Yarime, 2010)

(Göktepe-Hultén, 2010), (Hülsbeck, Lehmann \& Starnecker, 2013)

(Muscio, 2013), (Hemmert, Bstieler \& Okamuro, 2014)

(Montoya \& Aguilar, 2013)

(Soh \& Subramanian, 2014)

(Bruneel et al., 2010), (Plewa, Korff, Baaken, \& Macpherson, 2013), (Hemmert, Bstieler \& Okamuro, 2014)

(Bodas Freitas, Marques \& Silva, E. M. D. P. E., 2013)

(Ankrah \& AL-Tabbaa, 2015)

(Dueñas \& Duque, 2015)

(Muscio \& Pozzali, 2013)

(Al-Tabbaa \& Ankrah, 2016)

(Bruneel, D’esteb \& Salter, 2010) (Bjerregaard, 2010)

(Balconi \& Laboranti, 2006)

La relación entre académicos e industriales es parte fundamental para la RUE. A este respecto se ha encontrado que los investigadores académicos interactúan con la industria por diversos canales. Los canales más utilizados son la consultoría, el contrato de investigación, la investigación conjunta y la capacitación, en contraste con las actividades derivadas de la salida de 
patentes o actividades de spin out (D’este \& Patel, 2007). Dicha interacción puede ser muy firme o muy débil. Las conexiones fuertes se relacionan con el alto rendimiento científico y con las colaboraciones del personal de frontera, debido a la proximidad cognitiva y a las relaciones personales (Balconi \& Laboranti, 2006).

Por otro lado, las diferencias culturales que existen pueden debilitar la interacción, complicando el intercambio de conocimientos y el control de los proyectos de colaboración. Especialmente si están mal dirigidos. La debilidad de la interacción es particularmente notable en la colaboración entre la micro y pequeña industria y los investigadores de las instituciones públicas (Bjerregaard, 2010).

A pesar de las diferencias, los investigadores pueden llegar a tener puntos de encuentro comunes para el intercambio de conocimientos y la comunicación de sus proyectos conjuntos (Bjerregaard, 2010). Estos espacios comunes podrían y deberían incluir a los empresarios, ya que las habilidades sociales de algunos investigadores constituyen un recurso muy valioso para llenar los vacíos institucionales percibidos en las prácticas de I+D con la industria (Muscio \& Pozzali, 2013).

A pesar de contar con las cualidades individuales por parte de los investigadores, la distancia cognitiva entre las universidades y empresas dificulta significativamente la frecuencia de las interacciones entre ambos organismos. Un recurso con potencial para mejorar el acercamiento entre la universidad y la industria será la comunicación cara a cara (Balconi \& Laboranti, 2006). Así mismo, inducir una alta inteligencia emocional en la relación entre el empresario y el investigador y una alta empatía, podrían agregar ventajas importantes en la vinculación entre el binomio E-I; por ejemplo, la capacidad de liderazgo, el espíritu innovador y un manejo eficaz de la tensión en proyectos de I+D (Humphrey, 2013). Se sabe, también, que los motivos de los investigadores y los empresarios son la búsqueda del bienestar, sin interés por tener el control sobre los demás. A pesar de que los beneficios de los resultados de la transferencia de conocimientos son para cada organización individual, estos beneficios no llegan a la sociedad, como se menciona comúnmente al buscar el apoyo del gobierno (Ankrah, Burgess, Grimshaw \& Shaw, 2013).

La RUE se conoce poco. Sus potenciales no se han explorado del todo en países emergentes. Se aprecia una desintegración de los resultados de las investigaciones y la gestión de las colaboraciones carecen de visión global (Philbin, 2008), (Ankrah \& AL-Tabbaa 2015).

El enfoque humano entre el binomio Empresario-Investigador (E-I), se ha venido ignorando. Las diferencias y características del personal involucrado no se conocen del todo (Azagra-Caro, 2007). En la realidad actual, el investigador y el empresario no interactúan ni se retroalimentan 
directamente como se esperaría para un sistema eficaz de innovación. Por diversos factores, el entendimiento y la colaboración entre el académico y el industrial siguen siendo difíciles (Martínez \& Ikertia, 2013).

El presente estudio se basa en el binomio E-I. Este binomio, es la clave en el contexto de la RUE. El empresario y el investigador son los que pueden resolver los problemas causados por las políticas ineficaces, la falta de estructura y los errores de dirección (Bjerregaard, 2010). El binomio E-I no cuenta con las condiciones adecuadas para realizar proyectos conjuntos de I+D y TTC con mayor frecuencia.

El enfoque de esta investigación es la interacción humana entre empresario e investigador, pues se infiere la existencia de una brecha entre ambos (BEI). Se estima que esta brecha está impidiendo un acercamiento “cara-a-cara” y eficaz entre E-I, lo que obstaculiza el desarrollo de un mayor número de proyectos de $\mathrm{I}+\mathrm{D}$ en colaboración conjunta. También se piensa que la empatía entre los actores puede tener un papel importante en la frecuencia de la colaboración y en la calidad de la misma. Además, se infiere que no todos los factores que afectan la RUE desde el punto de vista organizacional, influyen de la misma manera en el nivel del binomio E-I por lo cual las políticas resultan ineficaces en muchos casos.

Este trabajo está dirigido a confirmar la existencia de la BEI, entender la importancia de la empatía en la colaboración conjunta entre E-I y determinar qué factores afectan la relación entre ambos. El resultado proporcionará una visión alternativa para mejorar las políticas al conocer la realidad de los actores principales en el sistema de innovación.

Se presentan en este artículo los avances encontrados sobre la percepción del investigador respecto a la existencia de una brecha entre el empresario y el investigador (BEI). También se presenta el nivel de importancia que el investigador da a la empatía para la realización de trabajos conjuntos entre E-I. Por último, se muestra la percepción de los investigadores sobre cuáles de los factores que afectan la RUE influyen más significativamente en la relación personal entre E-I para la colaboración conjunta en proyectos de I+D y TTC. Al final se listan factores que los investigadores encuestados perciben como importantes para mejorar la relación entre E-I.

\section{Método}

La metodología aplicada para el estudio fue de tipo cualitativa, transversal con un alcance exploratorio, descriptivo. La recolección de los datos se realizó mediante cuestionarios. La muestra fue de 25 individuos, todos investigadores de instituciones de educación superior (IES) o centros de investigación, sin distinción entre públicos o privados, pero con experiencia en colaboración conjunta en proyectos de I+D o TTC. Al final, 
se obtuvieron 25 encuestas respondidas. El estudio se realizó en México, en un periodo de tiempo entre enero y junio del 2016.

El procedimiento diseñado fue el siguiente:

1. Se tomó como base una lista de elementos que facilitan o impiden las relaciones entre la empresa y la universidad, de Ankrah y AL-Tabbaa (2015).

2. A dichos elementos, se agregaron otros considerados importantes por la experiencia en el campo, con lo que se generó una lista de 52 indicadores. Esta lista se redujo a 48 al final del proceso de depuración, tras la prueba piloto del test.

3. Los 48 indicadores, se reorganizaron por reclasificación como factores Cognitivos, Afectivos, y Situacionales (CAS), tomando como base la teoria de la empatia para las relaciones humanas (Fernández-Pinto, LópezPérez \& Márquez, 2008). Como resultado de la reclasificación, se obtuvieron tres bloques de factores (ver Tabla 2).

Tabla 2. Factores clasificados y codificados bajo el orden de cognitivos, afectivos y situacionales

(CAS) para evaluar la relación empresario-investigador en el contexto de la I+D y TTC

Factores cognitivos (FC)

C1-Capacitación

C2-Liderazgo

C3-Trabajo en equipo

C4-Capacidad de negociación

C5-Flexibilidad de adaptación

C6-Habilidad de trascender los límites

C7-El prestigio

C8-Nivel de conocimiento de

las capacidades de investigación

C9-Sinergia entre los actores

C10-Sensibilidad en TTC

C11-Grado de estudios*

C12-Pertenecer a una

institución o empresa reconocidos*
Factores afectivos (FA)

A1-Manejo de la confidencialidad

A2-Responsabilidad moral

A3-La confianza mutua

A4-La congruencia de

palabras y acciones*

A5-El riesgo de

investigación

A6-La capacidad de resiliencia

A7-La ética*

A8-Saber escuchar*

A9-El regionalismo*

A10-El compromiso mutuo

A11-La frecuencia de convivencia*

A12-La actitud*

A13-El trato respetuoso* A14-La comunicación
Factores situacionales (FS)

S1-Recursos adecuados

S2-Planes de incentivos

S3-Limitaciones de capacidad de las PyMe

S4-Políticas flexibles

S5-Estabilidad corporativa

S6-Gestión de proyectos

S7-Cultura organizacional

S8-Estructura de la organización

S9-Tamaño de la empresa

S10-La capacidad de absorción

S11-Movilidad e intercambio del personal

S12-Política, legislación y regulación

S13-Soporte y fomento para TTC

S14-Uso de intermediarios (OTTs)

S15-La proximidad geográfica

S16-Mecanismos y marcos jurídicos

internos y externos viables en el proceso

S17-Apoyo económico para innovación

S18-Equilibrio y equidad en la participación

S19-La burocracia del proceso de TTC*

S20-Contratos de licenciamiento preferenciales*

S21-El tiempo de respuesta*

S22-Costos del proceso de TTC

Fuente: Elaboración basada en: (Ankrah \& AL-Tabbaa, 2015).

Nota: Los factores marcados con $(*)$ son aportaciones de acuerdo a la experiencia de los autores. 
4. Para cada uno de los factores de la Tabla 2, se aplicó una escala de Likert de cinco niveles para medir la actitud del investigador sobre cuáles de estos factores influyen más desde el punto de vista de su relación “cara a cara” para la colaboración conjunta. Las categorías asignadas a la escala fueron: Totalmente de acuerdo (TA), De acuerdo (A), Neutral (N), En desacuerdo (D), Totalmente en desacuerdo (TD).

Para determinar la importancia que tiene la empatía para la participación en proyectos conjuntos desde la perspectiva del binomio E-I, se formuló la pregunta a):

a) ¿La empatía en la relación entre el empresario y el investigador es importante para la participación en proyectos conjuntos de I+D y TTC?

Para conocer la percepción del E-I sobre la existencia de una BEI, se formuló la pregunta b):

b) ¿Opina Usted que se percibe una brecha entre el empresario y el investigador en el contexto de la I+D y TTC?

Para abrir la oportunidad al E-I de aportar su visión personal y su experiencia sobre nuevos factores que afectan a la relación empresario investigador en proyectos conjuntos, se formuló la pregunta c):

c) ¿Qué otro factor o factores considera que afecta la relación entre el empresario y el investigador en el contexto de la I+D y TTC?

5. Con los tres bloques de factores y las tres preguntas descritas arriba, se diseñó el test que se aplicó a los investigadores.

6. Se realizó una prueba piloto a 7 investigadores, lo que permitió la depuración del test y la estimación de la fiabilidad de consistencia interna del instrumento, mediante el cálculo del coeficiente Alpha de Cronbach y el paquete estadístico SPSS.

7. Se obtuvo un Alpha de Cronbach = 0.98 de consistencia interna, por lo que se consideró aceptable el instrumento de acuerdo con CampoArias y Oviedo (2008).

8. Se seleccionó una muestra en base a expertos por combinación de: casos tipo, conveniencia y oportunidad (Hernández, Fernández \& Baptista, 2010).

9. Se aplicó el test por correo electrónico, con reforzamiento vía telefónica y en los casos posibles, se aplicó por entrevista.

\section{Resultados}

Sobre la Empatía

La Gráfica 1 muestra los resultados respecto a la importancia de la empatía entre el binomio E-I para su participación en proyectos conjuntos de I+D y TTC. Los datos muestran que, el 22\% de los investigadores encuestados piensa que la empatía es lo más importante para la participación en proyectos conjuntos de I+D y TTC y 56\% de los 
investigadores encuestados piensa que la empatía es muy importante para la participación en proyectos conjuntos de I+D y TTC. Es decir un 78\% de los investigadores encuestados percibe a la empatía como una variable muy significativa para la realización de proyectos conjuntos de I+D y TTC.

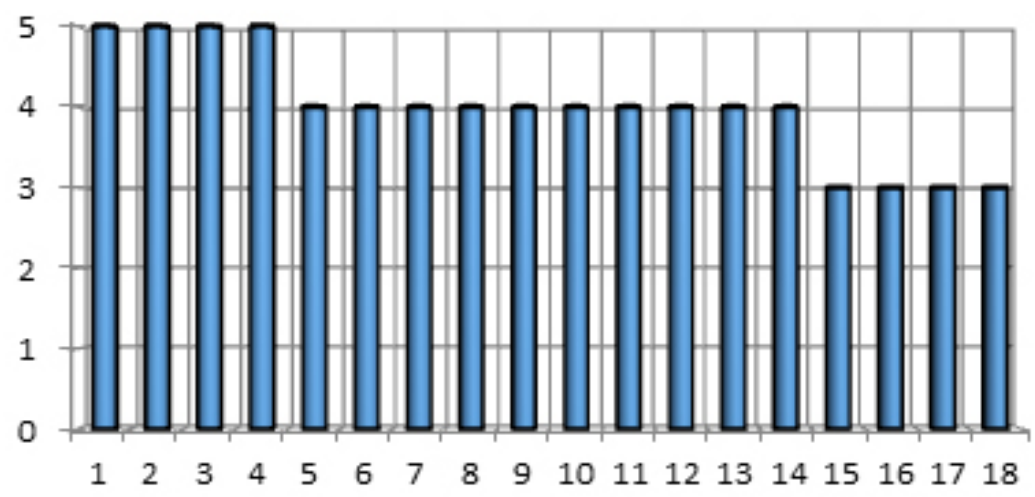

Gráfica 1. La importancia de la empatía entre E-I. El eje horizontal muestra la cantidad de respuestas totales obtenidas. El eje vertical muestra los valores asignados a los niveles de respuesta con lo más importante $(\mathrm{LMI})=5$ y Nada importante $(\mathrm{Ni})=1$.

\section{Sobre la BEI}

La Gráfica 2 presenta los resultados respecto a la existencia de una brecha entre el empresario y el investigador (BEI). Los datos muestran que el 75\% de los investigadores encuestados perciben la existencia de una BEI en el contexto de la colaboración conjunta para proyectos de I+D y TTC.

\section{La existencia de una BEI}

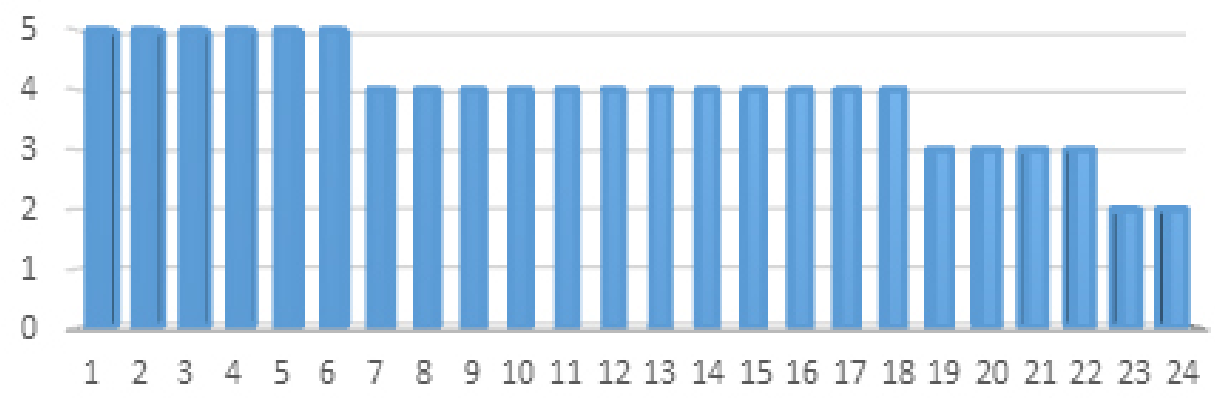

Gráfica 2. El eje horizontal muestra el total de respuestas obtenidas y el eje vertical los valores asignados a la escala con totalmente de acuerdo $(\mathrm{TA})=5 \mathrm{y}$ totalmente en desacuerdo $(\mathrm{TD})=1$. 


\section{Influencia de los factores CAS}

\section{Factores Cognitivos}

De los datos obtenidos para conocer cuáles de los factores que afectan las RUE influyen significativamente en la relación entre E-I, podemos ver que solo el $25 \%$ de los factores cognitivos impactan significativamente en la relación E-I, tomando en cuenta los promedios de los valores asignados por los investigadores (ver Gráfica 3).

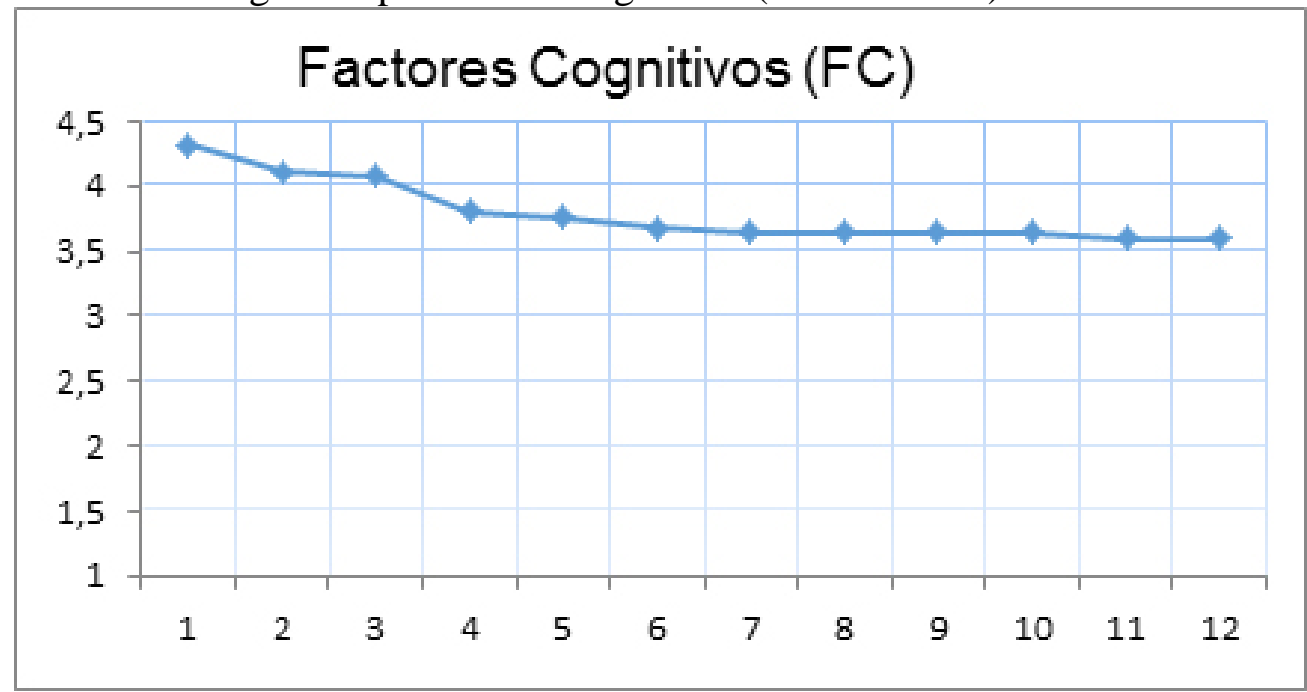

Gráfica 3. Promedio de los factores cognitivos que afecta la relación E-I, según la percepción de los investigadores.

En La Gráfica 4 se presentan los elementos con mayor puntuación (min=1, máx $=5)$ en función de su valor, percibido por los investigadores encuestados, destacando trabajo en equipo (C3) como el factor cognitivo más significativo para la relación E-I.

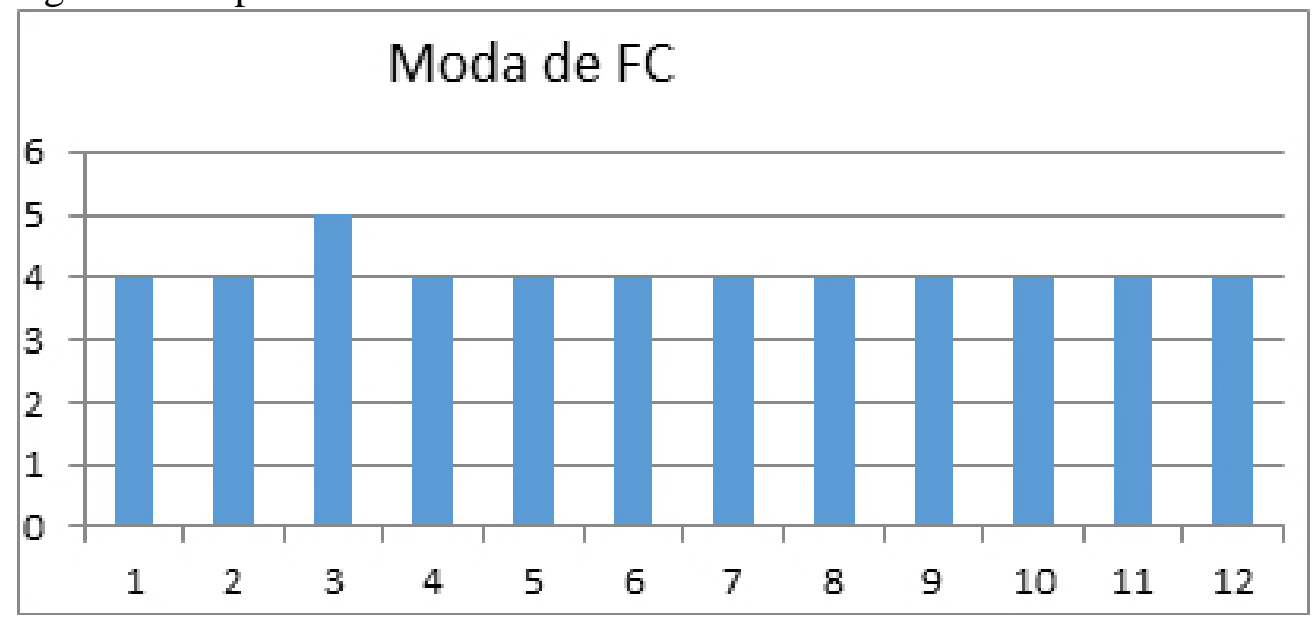

Gráfica 4. La moda de los factores cognitivos. 


\section{Factores Afectivos}

La Gráfica 5 muestra que, en promedio, el $57.14 \%$ de los factores afectivos impactan en la relación entre el empresario y el investigador para los proyectos conjuntos de I+D y TTC, de acuerdo con la percepción de los investigadores encuestados.

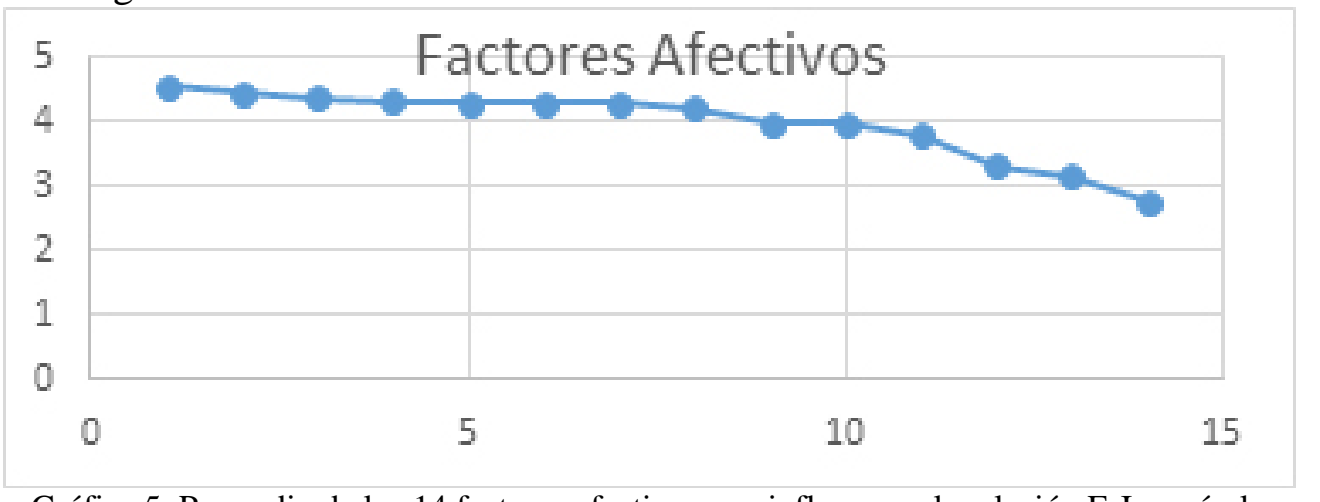

Gráfica 5. Promedio de los 14 factores afectivos que influyen en la relación E-I, según la percepción de los investigadores.

La Gráfica 6 muestra los factores afectivos que tuvieron la mayor valoración $(\mathrm{TD}=5)$ de los encuestados, respecto a su impacto en la relación E-I. Dichos factores afectivos son: manejo de la confidencialidad (A1), saber escuchar (A8) y el compromiso mutuo (A10).

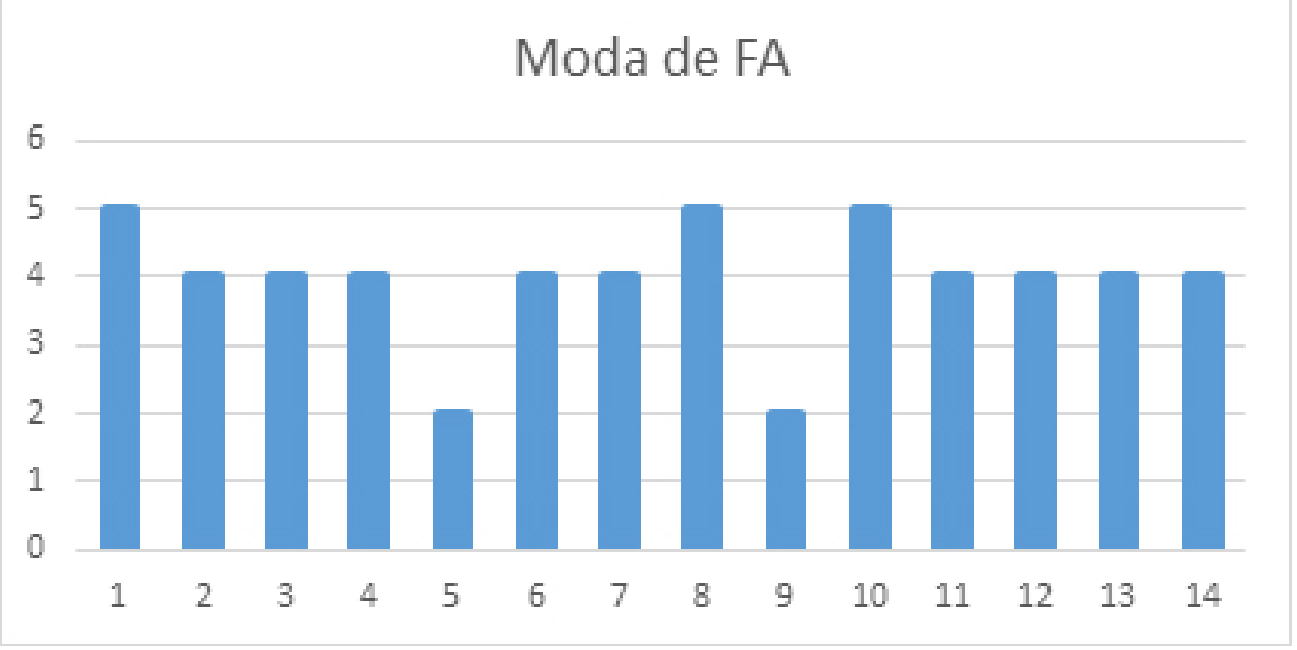

Gráfica 6. La moda de factores afectivos.

\section{Factores situacionales}

La Gráfica 7 muestra que el $41 \%$ de los factores situacionales influyen en el acercamiento del empresario y el investigador. 


\section{Factores Situacionales (FS)}

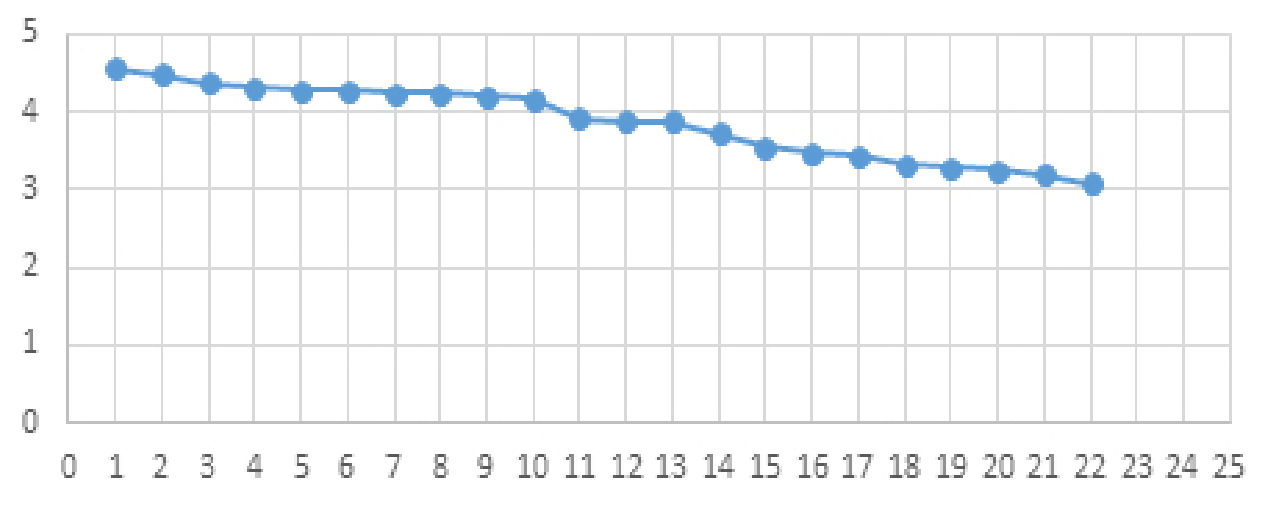

Gráfica 7. Promedio de los 22 factores situacionales que afecta la relación E-I, según la percepción de los investigadores.

La Gráfica 8 muestra los factores situacionales más significativos para la relación E-I, de acuerdo a la percepción de los investigadores encuestados. Dichos factores son: recursos adecuados (S1), movilidad e intercambio del personal (S11), la burocracia del proceso de TTC (S19) y el tiempo de respuesta (S21).

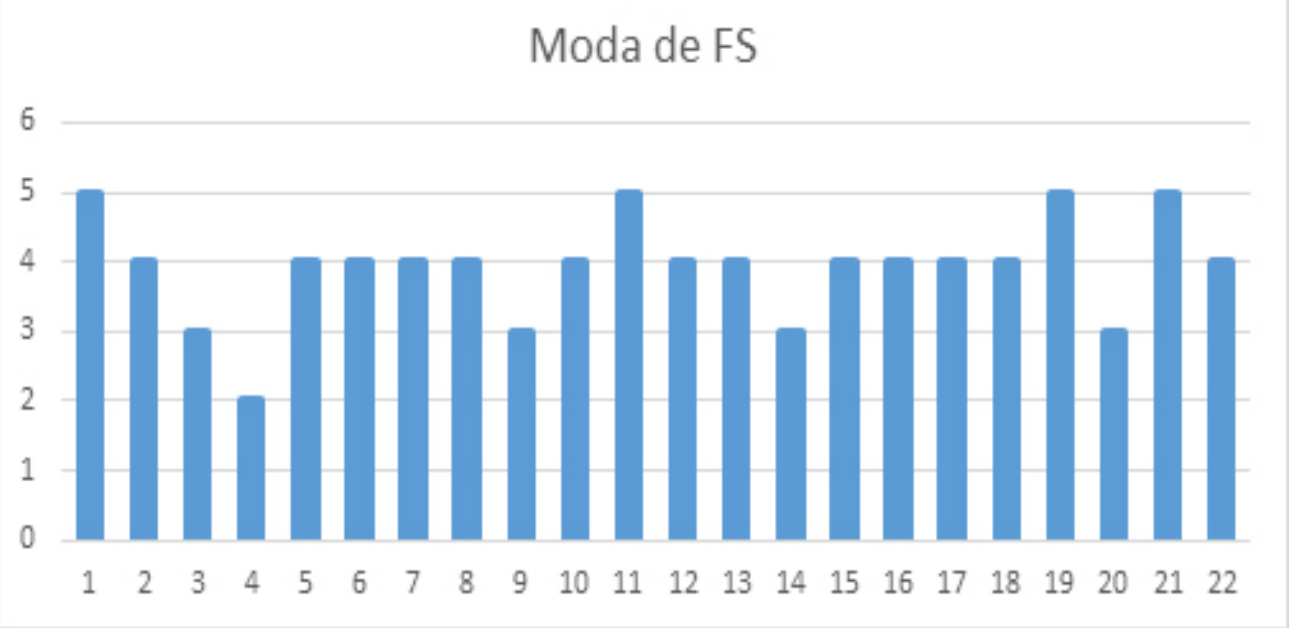

Gráfica 8. La moda de los factores situacionales.

La Tabla 3 muestra los factores cognitivos, afectivos y situacionales que más influyen en el acercamiento del binomio E-I para la colaboración conjunta en proyectos de I+D y TTC, de acuerdo a la puntuación dada por los investigadores encuestados, según su percepción. 
Tabla 3. Factores CAS que más influyen en la relación entre el empresario y el investigador de acuerdo a la opinión del investigador

Tipo de factor

Cognitivo

Afectivo

Situacional
Factor(es)

C3-Trabajo en equipo

A1-Manejo de la confidencialidad

A8-Saber escuchar

A10-El compromiso mutuo

S1-Recursos adecuados

S11-Movilidad e intercambio del personal

S19-La burocracia del proceso de TTC

S21-El tiempo de respuesta

\section{Sobre otros factores}

Los investigadores encuestados expusieron los factores que afectan a la relación del binomio E-I para la colaboración en proyectos conjuntos de I+D y TTC (ver Tabla 4).

Tabla 4. Factores que afectan la relación entre el empresario y el investigador para la colaboración en proyectos conjuntos de I+D y TTC, expresados por los investigadores encuestados

Prevalencia de intereses personales sobre los del equipo y los objetivos del proyecto.

Falta de consenso en el concepto de innovación.

Poco entendimiento mutuo del rol y contexto de cada uno (empresario e investigador).

Falta de educación empresarial sobre innovación para docentes e investigadores.

Escasa o nula comunicación directa con el interesado durante el proyecto.

Falta de mecanismos para fácil contacto con el investigador.

Escasa o nula promoción de reuniones entre Empresarios e Investigadores.

Fomento a relaciones E-I más estrechas.

Desconfianza de la empresa de dar a conocer su ventaja competitiva.

Falta de visión del empresario sobre el contexto de la investigación (tiempos, alcances, estructura, burocracia y limitaciones).

Falta hacer más eficiente la apertura entre la universidad y la empresa.

Foros y medios de divulgación ineficaces o inadecuados que producen malas experiencias para ambos.

Sensación de programas y sistemas fraudulentos (Se requiere evaluar los resultados y reestructurar los programas en mejora continua).

El dinero que quitan las instituciones al investigador por las ganancias de los proyectos.

El costo del proyecto para el empresario.

El financiamiento que el empresario tiene que otorgar para la investigación.

La falta de claridad sobre el costo beneficio antes de iniciar el proyecto. (Falta de estudios de mercado y de fondos para realizarlo).

Falta de planeación, coordinación y seguimiento de las tareas de los proyectos. (No existe una buena gestión del tiempo).

Intervención de terceros entre el empresario y el investigador para la realización del proyecto. 


\section{Conclusión y discusión}

En las últimas dos décadas se han realizado diversos estudios para mejorar las relaciones entre la universidad y la industria, así como para entender las características de los diferentes actores y la manera en que interactúan. Se han encontrado una gran cantidad de factores muy importantes (concentrados en 48 en este trabajo) y situaciones que afectan la RUE. Sin embargo, la interacción entre el empresario y el investigador ha sido poco estudiada desde el punto de vista personal. La intención de este trabajo fue acercar un poco la lupa y ver qué está pasando entre este binomio E-I en el contexto de la colaboración conjunta. En primer lugar, los resultados obtenidos respaldan la existencia de una brecha entre el empresario y el investigador, ya que el $\mathbf{7 5 \%}$ de los investigadores encuestados perciben la existencia de una BEI en el contexto de la colaboración conjunta para proyectos de I+D y TTC. Este hecho permite abrir una línea de investigación que lleve a entender la razón o las razones de que exista esta brecha, a pesar de los esfuerzos que se hacen para mejorar las relaciones entre la universidad y la industria. La hipótesis aquí sería que, los esfuerzos para mejorar las RUE no están tomando en cuenta las verdaderas necesidades que tiene el binomio E-I para lograr una colaboración conjunta eficiente y eficaz. Por tanto, las políticas y modelos de vinculación no pueden ser tan eficientes y eficaces como se espera.

En segundo lugar, se tiene la certidumbre de que la empatía es un factor muy importante a considerar en las actividades que promueven la vinculación y los proyectos de colaboración entre empresarios e investigadores, ya que el $\mathbf{7 8 \%}$ de los investigadores encuestados percibe a la empatía como una variable muy significativa para la realización de proyectos conjuntos de I+D y TTC. Si se aplica esta visión al desarrollo de programas de fomento a la vinculación entre universidad e industria, podrían incluirse elementos que gestionen el aumento de la empatía entre el empresario y el investigador.

En tercer lugar, se ha podido demostrar que un cambio de enfoque sobre los factores que afectan la RUE, revela una realidad distinta si se ve desde el nivel del binomio E-I. Las entrevistas con los investigadores revelan situaciones adversas, tales como: la incongruencia de las políticas externas de vinculación con la realidad cotidiana del investigador, la falta de condiciones para la retroalimentación cara a cara con el empresario y la escases de recursos, una comunicación escabrosa entre empresario, investigador y gobierno, además de la falta de retribución significativa para los que se aventuran a la colaboración conjunta de proyectos de I+D y TTC. Estas situaciones han generado descontento, desmotivación, indiferencia y apatía. Del mismo modo, se pudo comprobar que estos problemas tienen décadas y, aunque se han ido buscando mejoras, hoy en día siguen formando 
barreras que frenan el desarrollo necesario del sistema de innovación a los niveles de competitividad global requeridos. Los resultados respaldan el sentir de los investigadores, ya que de los factores que afectan a la empresa y a la universidad desde el enfoque organizacional, solo el 16.66\% influye más significativamente en la relación entre el empresario y el investigador desde el enfoque humano. Si aplicáramos el Principio de Pareto para la calidad a esta situación (pocos vitales muchos triviales), podríamos decir que una mejora sustancial en la calidad de las relaciones entre el empresario y el investigador se lograría adecuando los modelos y las políticas organizacionales o institucionales (tanto internas como externas) para que en conjunto satisfagan las necesidades del binomio E-I, en cuanto a esos factores realmente relevantes. Con lo anterior, el sistema de innovación se estaría dirigiendo con más rapidez a la eficiencia y eficacia requerida para la competitividad global, gracias a un pensamiento centrado en el binomio E-I, teniendo como diana el mejoramiento de la calidad de vida de la sociedad.

Los empresarios, por su parte, podrían tener varias coincidencias con los investigadores, sin embargo, la naturaleza de su cultura empresarial implica la existencia de elementos complementarios para el acercamiento del binomio E-I, los cuales no se han esclarecido aun y requieren de investigación. Los resultados permitirían contribuir a que se reduzca la BEI y se incremente el potencial del binomio E-I, así como sus deseos de realizar colaboración conjunta, creando un ecosistema propicio para su constante encuentro, interacción e innovación. Los beneficios se reflejarán en más soluciones a los problemas sociales de base tecnológica, mayor productividad, patentamiento y competitividad.

\section{References:}

1. Al-Tabbaa, O. \& Ankrah, S. (2016) Social capital to facilitate 'engineered' university-industry collaboration for technology transfer: A dynamic perspective. Technological Forecasting \& Social Change 104 1-1

2. Ankrah S., \& AL-Tabbaa O. (2015) Universities-industry collaboration: A systematic review. Scandinavian Journal of Management 31, 387-408

3. Ankrah, S. N., Burgess, T. F., Grimshaw, P. \& Shaw, N. E. (2013). Asking both university and industry actors about their engagement in knowledge transfer: What single-group studies of motives omit. Technovation, 33, 50-65.

4. Azagra-Caro, J. M. (2007). What type of faculty member interacts with what type of firm? Some reasons for the delocalisation of university-industry interaction. Technovation, 27, 704-715. 
5. Balconi, M. \& Laboranti, A. (2006). University-industry interactions in applied research: The case of microelectronics. Research Policy, 35(10), 1616-1630.

6. Bekkers, R. \& Bodas Freitas, I. (2008). Analysing knowledge transfer channels between universities and industry: To what degree do sectors also matter? Research Policy, 37, 1837-1853.

7. Bjerregaard, T. (2010). Industry and academia in convergence: Microinstitutional dimensions of R\&D collaboration Technovation, 30, 100-108.

8. Bodas Freitas, I. M., Marques, R. A. \& Silva, E. M. D. P. E. (2013). University-industry collaboration and innovation in emergent and mature industries in new industrialized countries. Research Policy, 42, 443-453.

9. Bruneel, J., d'Este, P. \& Salter, A. (2010). Investigating the factors that diminish the barriers to university-industry collaboration. Research Policy, 39(7), 858-868.

10. Campo-Arias, A. \& Oviedo,H. (2008). Psychometric properties of a scale: internal consistency. Revista de salud pública. 10 (5):831-839.

11. Chen, D.H. \& Dahlman, C.J. (2005). The knowledge economy, the KAM methodology and World Bank operations. World Bank Institute. Working Paper.

12. D'este, P. \& Patel, P. (2007). University-industry linkages in the UK: What are the factors underlying the variety of interactions with industry? Research Policy, 36, 1295-1313

13. De Fuentes, C., \& Dutrenit, G. (2012). Best channels of academiaindustry interaction for long-term benefit. Research Policy, 41(9), 1666-1682.

14. Dueñas Quintero, D. M. \& Duque Oliva, E. J. (2015). Calidad de las relaciones universidad-empresa: un análisis desde el enfoque de marketing relacional en Boyacá. Pensamiento \& Gestión, (38), 147175.

15. Dutrenit, G. \& Arza, V. (2010). Channels and benefits of interactions betwee public research organisations and industry: comparing four Latin American countries. Science and Public Policy, 37 pp. 541-553

16. Etzkowitz, H. \& Leydesdorff, L. (2000). The dynamics of innovation: from National Systems and "Mode 2" to a Triple Helix of universityindustry-government relations. Research policy, 29(2), 109-123.

17. Fernández, A. C., De Souza, B. C., da Silva, A. S., Suzigan, W., Chaves, C. V. \& Albuquerque, E. (2010). Academy-industry links in Brazil: evidence about channels and benefits for firms and researchers. Science and Public Policy, 37(7), 485-498 
18. Fernández-Pinto, I., López-Pérez, B. \& Márquez, M. (2008). Empatía: Medidas, teorías y aplicaciones en revisión. Anales de psicología, 24(2), 284-298.

19. Göktepe-Hultén, D. (2010). University-industry technology transfer: who needs TTOs? International journal of technology transfer and commercialization, 9 (1-2), 40-52.

20. Hemmert, M., Bstieler, L. \& Okamuro, H. (2014). Bridging the cultural divide: Trust formation in university-industry research collaborations in the US, Japan, and South Korea. Technovation, 34, 605-616.

21. Hernández Sampieri, R., Fernandez Collado, C. \& Baptista Lucio, P. (2010). Metodología de la investigación. México: McGraw-Hill/ Interamericana editores S.A. de C.V.

22. Hülsbeck, M., Lehmann, E. E. \& Starnecker, A. (2013). Performance of technology transfer offices in Germany. The journal of technology transfer, 38(3), 199-215.

23. Humphrey, R. H. (2013). The benefits of emotional intelligence and empathy to entrepreneurship. Entrepreneurship Research Journal, 3(3), 287-294.

24. Inzelt, A. (2004). The evolution of university-industrygovernment relationships during transition. Research Policy, 33, 975-995.

25. Martínez, R. \& Ikertia, F. (2013). La relación universidad-empresa en bizkaia: Un enfoque cualitativo y propuesta de mejora. Revista EAN, (61), 123-126.

26. Montoya Ríos, F. J. \& Aguilar Zambrano, J. J. (2013). La Relación Universidad-Empresa en las Prácticas Empresariales: un Modelo Conceptual desde las Técnicas de Generación de Ideas. Journal of technology management \& innovation, 8, 47-47.

27. Motohashi, K. (2005). University-industry collaborations in Japan: The role of new technology-based firms in transforming the national innovation system. Research Policy, 34, 583-594.

28. Muscio, A. (2013). University-industry linkages: What are the determinants of distance in collaborations? Papers in Regional Science, 92, 715-739.

29. Muscio, A. \& Pozzali, A. (2013). The effects of cognitive distance in university industry collaborations: Some evidence from Italian universities. Journal of Technology Transfer, 38, 486-508.

30. Philbin, S. (2008). Process model for university-industry research collaboration. European Journal of Innovation Management, 11, $488-521$. 
31. Plewa, C., Korff, N., Baaken, T. \& Macpherson, G. (2013). University-industry linkage evolution: An empirical investigation of relational success factors. R\&D Management, 43, 365-380.

32. Radu, L. D. (2015). Investments In Technological Innovations: A Literature Review Of Organization Determinants. European Scientific Journal.

33. Sapién-Aguilar, A. L., Piñón-Howlet, L. C. \& Gutiérrez-Diez, M. D. C. (2015). Relaciones reciprocas en el modelo de triple hélice mediante variables de interacción. European Scientific Journal, 11(20).

34. Shichijo, N., Baba, Y. \& Yarime, M. (2010). Sources of success in advanced materials innovation: The role of "core researchers" in university - industry collaboration In Japan. International Journal of Innovation Management, 14, 201-219.

35. World Economic Forum (2011). The global competitiveness report 2011-2012. Geneva:WEF 